\title{
COVID-19: An effective vaccine is needed
}

\author{
Lisset Hermida and Ricardo Silva
}

A

novel coronavirus named SARS-CoV-2 is responsible for the present pneumonia outbreak called COVID-19 that started in early December 2019 in Wuhan City, Hubei province, China ${ }^{1}$. Until March 12, SARS-CoV-2 had caused 80980 infections and 3173 deaths in China, but even worst, it is currently spreading fast in other countries, reaching 45293 infections and 1460 deaths outside China, being the most affected countries Italy, Iran and South Korea².

New cases identified in Wuhan are thought to have acquired the infection from a zoonotic source, as many reported visiting or working in the Huanan Wholesale Seafood Market. The coronaviruses are naturally hosted and evolutionarily shaped by bats ${ }^{3,4}$. The whole-genome sequence identity of the SARSCoV-2 has $96.2 \%$ similarity to a bat SARS-related coronavirus (SARSr-CoV; RaTG13) collected in Yunnan province, China ${ }^{5,6}$. Nevertheless, this percentage indicates that an intermediate species should be responsible for the transmission to humans.

The incredibly rapid and effective response from the Chinese government in managing the virus widespread transmission resulted in the creation of a comprehensive epidemiological strategy that has led to the nature of newer diagnostic tools as well as a more advanced clinical management. On top of that, all scientific research has been primarily focused on the virus and its outbreak, allowing China, in a record time of just three months, to gather groundbreaking findings to share with the scientific and health community worldwide. So far, we can identify the critical issues associated with the current outbreak. First, the high virus spreads based on direct and indirect forms of transmission. SARS-CoV-2 is transmitted through drops and fomites during close and unprotected contact between an infected person an uninfected one. Airborne spread of the virus has not been reported; however, it may be envisaged whether specific aerosol generation procedures are carried out in health care facilities ${ }^{7}$. Researchers are trying to relate the particular nature of the Spike protein and its interaction with the host cell to explain the high level of transmission ${ }^{8}$. Second, in a recent study involving 1099 patients in Wuhan province, China, fever was present in $43.8 \%$ of patients at the time of admission but developed in $88.7 \%$ during hospitalization ${ }^{9}$. This means that people infected with afebrile are abundant during the initial period of the disease and therefore favor the spread of the virus while escaping temperature control. Third, about $20 \%$ of confirmed patients develop severe clinical manifestations ${ }^{10}$. This situation is a challenge for any health system. Fourth, WHO has recently estimated a global fatality rate of $3.4 \%$, but this figure must be confirmed once the epidemic is over $^{11}$. As for China, until March 12, the estimated fatality rate of the outbreak was $3.9 \%{ }^{10}$.

The combination of the factors mentioned above indicates that we are facing an unprecedented respiratory virus infection. This is in line with the high level of required prevention and control measures implemented by China to reach an effective reduction in the number of daily new infected cases. Given the previous factors, as well as the current spreading of the virus across the globe (particularly in Italy, which is currently lockdown), the preventive vaccination appears to be the
DOI. 10.21931/RB/2020.05.02.1

most effective solution to contain the virus. Why is a vaccine against SARS-CoV-2 possible? Because the results obtained so far, either in preclinical or clinical trials with other two lethal Beta coronaviruses, can guide the development of a vaccine against SARS-CoV-2.12,13. It has been demonstrated the protective capacity of neutralizing antibodies and cell-mediated immunity in animal models ${ }^{14,15,16,17}$. On the other hand, the current success of plasma therapy from the cured COVID-19 patients indicates the functionality of antibodies against the SARS-CoV-2 replication ${ }^{18}$. Finally, the rapid DNA sequencing of several isolates of the SARS-CoV-2 by Chinese scientists, up to date over 100, has provided suitable information to design the vaccine candidate ${ }^{19}$.

Accordingly, up to now, there are at least 35 vaccine candidates under research and development from different laboratories worldwide 20 . Different approaches are being used, such as whole-inactivated, subunit vaccines, vectored vaccines, DNA, and RNAm vaccines, among others. Particularly in China, Dr. Zheng Zhongwei, Director of the Development Center for Medical Science and Technology from the National Health Commission, said that China has nine ongoing research projects on vaccine development, and some will hopefully enter clinical trials in April this year. The main types of vaccines that China is working on include inactivated, nucleic acid-based, and vector vaccines. The United States biotech company Inovio Pharmaceuticals and its partner Beijing Advaccine Biotechnology are developing a DNA vaccine called INO-480021. The vaccine candidate is based on DNA technology, which has been used for its GLS-5300 MERS coronavirus vaccine candidate. This vaccine candidate GLS-5300 showed the first positive results of immunogenicity in humans against MERS-CoV22. In January, another US biotech company GeoVax partnered with Chinese vaccine developer BravoVax to develop a vector vaccine, which uses the MVA platform ${ }^{21}$. In addition, the first reported inactivated vaccine candidate against SARS-CoV-2 is being developed by China's state-owned pharmaceutical enterprise Sinopharm Group, as reported by Chinese officials at a press conference on February 17.

Nevertheless, despite the urgent need, the National Medical Product Administration of China claims to pass the required tests for the vaccines to be administered in healthy humans. It has been recognized immunopathogenic events associated with the most severe cases of COVID-1923. Also, there is a possibility of antibody-dependent enhancement (ADE) since it has been described for the other two related lethal coronaviruses ${ }^{24}$. Regarding the two previous elements, the WHO already published the Synopsis for designing and conducting the vaccine clinical trials to prevent COVID-1925.

It is worth noting that COVID-19 is the third zoonosis related to human lethal coronaviruses. In 2002, a human pathogenic CoV, severe acute respiratory syndrome (SARS-CoV), caused 8,096 human infections with a 9.6\% mortality rate ${ }^{26}$. In 2012, the coronavirus Middle East respiratory syndrome MERS-CoV was another emerging pathogen that provoked outbreaks in the Arabian Peninsula and travelers from this region. As of April 24, 2016, a total of 1,728 cases with 624 dea- 


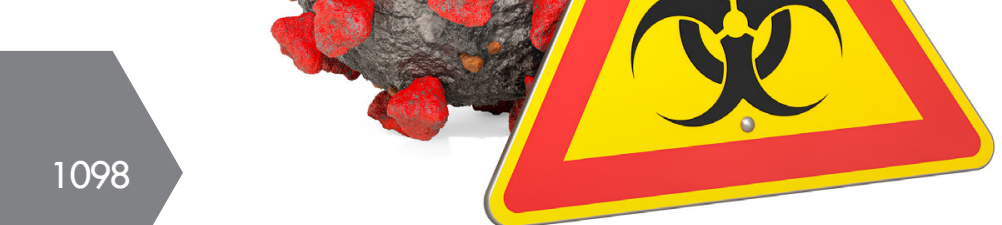

Figure 1. Biosafety Level 3 containment is required for culturing and characterizing the SARS-CoV-2.

ths (36.1\% mortality) were reported in 27 countries $^{27}$. As the human population continues to increase, demand for agricultural land grows, exposing livestock and humans to infections in the wild. Climate change is also modifying the ecosystem and the concentration of animal vectors, and rapid expansion in air traffic, the movement of people across different borders, political instability and conflicts mean these new pathogens can quickly spread across the world. Given this unfavorable scenario, the development of vaccines against SARS-CoV-2 should be accomplished with a strategic viewpoint. An effective vaccine should be able to protect not only against the three already known lethal coronaviruses, but also be able to prevent against another potentially fatal human Beta CoVs, which could cause similar outbreaks in the future. In fact, one Research Proposal based on this approach is being currently evaluated by the China Cuba Biotechnology Joint Innovation Center (CCBJIC), in the Yongzhou Municipality of Hunan Province, $\mathrm{China}^{28}$. The world should be ready to combat these proven events occurring in nature.

\section{Conclusions}

The entire globe is now facing a big challenge: the effective containment of the SARS-CoV-2 transmission. Based on China's experience so far, the implementation of stringent and rigorous control measures is currently the most successful way to reduce infections. However, given the critical issues related to the current outbreak and the economic differences of the world's countries, an effective vaccine will be the ultimate goal to stop the ongoing coronavirus transmission and next virus zoonosis.

\section{Bibliographic references}

1. Huang, C. et al. Clinical features of patients infected with 2019 novel coronavirus in Wuhan, China. Lancet 395, 497-506 (2020).

2. OCHA Iraq. Situation Reports. https://www.who.int/emergencies/ diseases/novel-coronavirus-2019/situation-reports/ (2009).

3. Cui, J., Li, F. \& Shi, Z. L. Origin and evolution of pathogenic coronaviruses. Nature Reviews Microbiology vol. 17 181-192 (2019).

4. Li, X., Song, Y., Wong, G. \& Cui, J. Bat origin of a new human coronavirus: there and back again. Sci. China Life Sci. 63, 461-462 (2020).

5. Zhou, P. et al. A pneumonia outbreak associated with a new coronavirus of probable bat origin. Nature 579, 270-273 (2020).

6. Paraskevis, D. et al. Full-genome evolutionary analysis of the nov- el corona virus (2019-nCoV) rejects the hypothesis of emergence as a result of a recent recombination event. Infect. Genet. Evol. 79, 104212 (2020).

7. WHO. WHO Director-General's opening remarks at the mission briefing on COVID-19 - 26 February 2020. 1-6 https://www. who.int/dg/speeches/detail/who-director-general-s-openingremarks-at-the-media-briefing-on-covid-19---3-march-2020 (2020).

8. Mallapaty, S. Why does the coronavirus spread so easily between people? Nature 579, 183-183 (2020).

9. Guan, W. et al. Clinical Characteristics of Coronavirus Disease 2019 in China. N. Engl. J. Med. NEJMoa2002032 (2020) doi:10.1056/NEJMoa2002032.

10.https://www.worldometers.info/coronavirus/.

11. WHO. WHO Director-General's opening remarks at the mission briefing on COVID-19 - 26 February 2020. 1-6 https://www. who.int/dg/speeches/detail/who-director-general-s-openingremarks-at-the-media-briefing-on-covid-19---11-march-2020 (2020).

12. Yong, C. Y., Ong, H. K., Yeap, S. K., Ho, K. L. \& Tan, W. S. Recent Advances in the Vaccine Development Against Middle East Respiratory Syndrome-Coronavirus. Front. Microbiol. 10, 1781 (2019).

13. Jiang, S., He, Y. \& Liu, S. SARS Vaccine Development - Volume 11 Number 7-July 2005 - Emerging Infectious Diseases journal CDC. doi:10.3201/EID1107.050219.

14.Liu, W. J. et al. T-cell immunity of SARS-CoV: Implications for vaccine development against MERS-CoV. Antiviral Research vol. 137 82-92 (2017).

15. Zhao, J. et al. Airway Memory CD4+ T Cells Mediate Protective Immunity against Emerging Respiratory Coronaviruses. Immunity 44, 1379-1391 (2016).

16. Chen, Z. et al. Recombinant Modified Vaccinia Virus Ankara Expressing the Spike Glycoprotein of Severe Acute Respiratory Syndrome Coronavirus Induces Protective Neutralizing Antibodies Primarily Targeting the Receptor Binding Region. J. Virol. 79, 2678-2688 (2005)

17. Muthumani, $K$. et al. A synthetic consensus anti-spike protein DNA vaccine induces protective immunity against Middle East respiratory syndrome coronavirus in nonhuman primates. Sci. Transl. Med. 7, (2015).

18. Potential treatments for novel coronavirus - Chinadaily.com. cn.https://covid-19.chinadaily.com.cn/a/202003/05/WS5e62126da31012821727d02f.html.

19. BIG Search - National Genomics Data Center - National Genomics Data Center. https://bigd.big.ac.cn/search?dbld=2019nCoVR\& $q=$ ncov\&page $=1$.

20.Cadila, Z., Vector, V., Vector, V. \& Vector, V. DRAFT landscape of COVID-19 candidate vaccines - 4 March. 4-6 (2020).

21. Teamwork underway to develop vaccine. https://enapp.chinadaily.com.cn/a/202003/12/AP5e69863ea3103a24b110a827.html.

22. Yoon, I. K. \& Kim, J. H. First clinical trial of a MERS coronavirus DNA vaccine. The Lancet Infectious Diseases vol. 19 924-925 (2019).

23. Huang, C. et al. Clinical features of patients infected with 2019 novel coronavirus in Wuhan, China. Lancet 395, 497-506 (2020).

24. Wan, Y. et al. Molecular Mechanism for Antibody-Dependent Enhancement of Coronavirus Entry. J. Virol. 94, (2019).

25. WHO I Coronavirus disease (COVID-2019) R\&D. WHO (2020).

26. Peiris, J. S. M., Guan, Y. \& Yuen, K. Y. Severe acute respiratory syndrome. Nature Medicine vol. 10 S88-S97 (2004).

27. Middle East respiratory syndrome coronavirus (MERS-CoV) Saudi Arabia. Saudi Med. J. 35, 1293 (2014)

28. https://oncubanews.com/en/cuba/cuba-and-china-to-open-biotechnological-innovative-center/ 